\title{
Finite $U$-induced competing interactions, frustration, and quantum phase transition in a triangular-lattice antiferromagnet
}

\author{
Avinash Singh* \\ Department of Physics, Indian Institute of Technology Kanpur - 208016
}

\begin{abstract}
The $120^{\circ}$ ordered antiferromagnetic state of the Hubbard model on a triangular lattice presents an interesting case of $U$-controlled competing interactions and frustration. The spin stiffness is found to vanish at $U_{\text {stiff }}^{*} \approx 6$ and the spin-wave energy $\omega_{M}$ at $\mathbf{q}_{M}=(2 \pi / 3,0)$ etc. is found to vanish at $U_{M}^{*} \approx 6.8$ due to competing spin couplings generated at finite $U$. The loss of magnetic order due to the magnetic instability at $\mathbf{q}_{M}$ yields a first-order quantum phase transition in the insulating state at $U=U_{\mathrm{M}}^{*}$. Implications of the quantum spin disordered insulator to the spin-liquid state and Mott transition in the organic systems $\kappa-(\mathrm{BEDT}-\mathrm{TTF})_{2} \mathrm{X}$ are discussed. Effects of hole and electron doping on magnetic ordering and spin stiffness are also examined.
\end{abstract}

\section{INTRODUCTION}

Recent ${ }^{1} \mathrm{H}$ NMR and static susceptibility measurements on the nearly isotropic, triangular-lattice antiferromagnet $\kappa-(\mathrm{BEDT}-\mathrm{TTF})_{2} \mathrm{Cu}_{2}(\mathrm{CN})_{3}$ have shown no indication of long-range magnetic ordering down to 32 $\mathrm{mK}$, well below the estimated exchange constant $J \sim 250$ $\mathrm{K}$, suggesting the realization of a quantum spin-liquid state. ${ }^{1}$ No signature of antiferromagnetic (AF) transition was seen in earlier EPR measurements as well. 2

On the other hand, a non-colinear $120^{\circ}$ ordered ground state is the accepted consensus for the $S=1 / 2$ quantum Heisenberg antiferromagnet (QHAF) on an isotropic triangular lattice ${ }^{3,4}$ Recent quantum Monte Carlo calculations yield a quantum reduction of $59 \%$ to the magnetic order from its classical value,,$\frac{5}{2}$ quite close to the spin-wave theory result of $52 \%$ in the first-order $1 / S$ expansion,,$\frac{6.7}{6 h i c h}$ is somewhat greater than the $40 \%$ reduction for the unfrustrated square-lattice.

In this paper we show that the absence of long-range magnetic order in the nearly isotropic organic antiferromagnet $\kappa-(\mathrm{BEDT}-\mathrm{TTF})_{2} \mathrm{Cu}_{2}(\mathrm{CN})_{3}$ can be understood in terms of strongly enhanced quantum spin fluctuations due to finite $U$-induced competing interactions and frustration. Indeed, for the half-filled Hubbard model on an isotropic triangular lattice, we find that in the insulating ordered state the spin stiffness vanishes at $U_{\text {stiff }}^{*} \approx 6$. The corresponding divergence in the spinfluctuation correction implies loss of long-range magnetic order at $U_{\text {order }}^{*}$ which is somewhat higher than $U_{\text {stiff }}^{*}$, yielding a finite- $U$ magnetic quantum phase transition in the insulating state. Even deeper in the insulating state, we find an instability of the $120^{\circ}$ ordered state at $U_{M}^{*}=6.8$ against out-of-plane spin fluctuations of wave vector $\mathbf{q}_{M}=(2 \pi / 3,0)$ etc., implying instability towards a F-AF state. Since spin fluctuations remain finite as $U \rightarrow U_{M}^{*}$, the magnetic instability implies a first-order quantum phase transition, thus pre-empting the vanishing spin-stiffness instability.

The realization in the triangular lattice of a nonmagnetic insulator state at intermediate $U$, in which magnetic ordering is suppressed by strong quantum spin fluctuations, is interesting as it allows, with decreasing
$U$, for a Mott-type metal-insulator transition not accompanied by any magnetic symmetry breaking. In this context, another quantum correction which assumes significance is that for the AF band gap. Indeed, two different scenarios emerge depending on the relative magnitudes of $U_{\text {order }}^{*}$ and $U_{\text {gap }}^{*}$, where the $120^{\circ} \mathrm{AF}$ order and AF band gap vanish, respectively. For $U_{\text {order }}^{*}>U_{\text {gap }}^{*}$, a non-magnetic insulator state lies between the paramagnetic metal (PM) and the AF insulator (AFI), whereas for $U_{\text {gap }}^{*}>U_{\text {order }}^{*}$, the magnetic transition is pre-empted, and there is a (nearly) first-order transition from AFI to $\mathrm{PM}$ at $U_{\text {gap }}^{*}$ when the two AF bands start overlapping, possibly with an intervening antiferromagnetic metallic (AFM) phase in a narrow $U$ range. Quantum corrections to quasiparticle dispersion and band gap due to motion of an added hole (electron) in the AF background, resulting in strong incoherence due to scrambling of the spin ordering, has been recently studied in detail for the $t-t^{\prime}$-Hubbard model on a square lattice ${ }^{8}$

The divergent quantum spin fluctuations due to vanishing spin stiffness and the magnetic instability towards a F-AF state may actually be a precursor to an exotic quantum spin-disordered state, as for the frustrated square-lattice antiferromagnet. Indeed, for the spinhalf $J-J^{\prime}$ Heisenberg model on a square lattice, or equivalently the strong-coupling $t-t^{\prime}$-Hubbard model, where the frustrating NNN coupling $J^{\prime}=4 t^{\prime 2} / U$ leads to vanishing spin stiffness and a $(0, \pi)$ instability towards a F-AF state at $J^{\prime} / J=t^{\prime 2} / t^{2}=0.5, \frac{9}{,}$ seriesexpansion studies ${ }^{10.11}$ of the ground-state energy indicate a continuous transition at $J^{\prime} / J \sim 0.4$ from the $\mathrm{AF}$ state which breaks spin-rotation symmetry to a columnar dimer (valence-bond-solid) state which breaks lattice translation symmetry, although it is controversial ${ }^{12}$ The location of the transition at $J^{\prime} / J \sim 0.4$ is surprisingly very close to where the AF order also vanishes ${ }^{9}$ Recently there has been strong interest in the critical theory of continuous quantum phase transitions between two phases with different broken symmetry, which requires going beyond the Landau-Ginzburg-Wilson paradigm 13

The suppression of magnetic ordering due to enhanced spin fluctuations is therefore also relevant in the layered system $\kappa-(\mathrm{BEDT}-\mathrm{TTF})_{2} \mathrm{Cu}\left[\mathrm{N}(\mathrm{CN})_{2}\right] \mathrm{Cl}$, which 
exhibits a genuine Mott transition not accompanied by any symmetry breaking 14 Generally, the organic systems $\kappa-(\mathrm{BEDT}-\mathrm{TTF})_{2} \mathrm{X}$, where $X$ denotes inorganic monovalent anion, have emerged as a new class of correlated electron systems exhibiting antiferromagnetism, superconductivity, and metal-insulator transition $\stackrel{15,16}{ }$ Recent discovery of superconductivity in $\mathrm{Na}_{\mathrm{x}} \mathrm{CoO}_{2} \cdot \mathrm{yH}_{2} \mathrm{O}^{17}$ and the observation of low-temperataure insulating phases in some $\sqrt{3}$-adlayer structures such as $\mathrm{K}$ on $\mathrm{Si}[111], 18$ have also renewed interest in correlated electron system on triangular lattices. As a recent example of quasitwo-dimensional antiferromagnetism on a triangular lattice exhibiting the $120^{\circ}$ spin ordering, $\mathrm{RbFe}\left(\mathrm{MoO}_{4}\right)_{2}$ has been studied using elastic neutron scattering, 19 and magnetic resonance and magnetization experiments. 20

The Hubbard model on a triangular lattice has been studied recently using a variety of tools. The nonmagnetic insulating state near the Mott transition has been studied using the path integral renormalization group method, ${ }^{21}$ in which the $\mathrm{HF}$ results are systematically improved to reach the true ground state by taking account of quantum fluctuations. Results show a generic emergence of a non-magnetic insulating state sandwiched by a Mott metal-insulator transition and an AF transition. The zero-temperature phase diagram has been studied using the slave boson technique and the exact diagonalization 22 The mean-field SB approach yields a rich phase diagram qualitatively resembling the $\mathrm{HF}$ results. ${ }^{23,24}$ One-electron density of states has been examined using the quantum Monte Carlo method,, 25 showing a pseudogap development for intermediate $U$, accompanied by two peaks in the spin structure factor, signaling the formation of a spiral spin density wave (SDW). A weak-coupling RG analysis applied to the anisotropic triangular lattice shows that frustration suppresses the antiferromagnetic instability in favour of a superconducting instability: ${ }^{26}$ A magnetic field induced exotic spin-triplet superconductivity has been proposed having strong ferromagnetic fluctuations. ${ }^{27}$ Ground-state spin structure of $\mathrm{Cr}$ and $\mathrm{Mn}$ monolayers on $\mathrm{Cu}[111]$, proposed as ideal candidates for physical realization of frustrated 2D itinerant antiferromagnets, has been investigated by performing $a b$ initio calculations based on the density-functional theory in the local spin-density approximation. ${ }^{28}$

A spin-liquid type non-magnetic insulating (NMI) state sandwiched between a weak-coupling PM state and a strong-coupling AFI state has also been obtained for the $t-t^{\prime}$-Hubbard model on a square lattice and an anisotropic triangular lattice using the path integral renormalization group method. ${ }^{29,30}$ The NMI state has been recently suggested to be a new type of degenerate quantum spin phase having gapless and dispersionless spin excitations ${ }^{30}$ At the same time, this result of an intervening NMI state is in contradiction to the earlier finding of an intermediate metallic antiferromagnetic state (AFM) 31 We briefly compare the two types of lattices to highlight the common and distinguishing features of the AF state.
Frustration suppresses the spin stiffness in both cases, rendering the $\mathrm{AF}$ state more susceptible to loss of magnetic order due to quantum fluctuations. While frustration is explicitly controlled by $t^{\prime}$ in the square lattice and can be tuned to zero, the triangular lattice provides a subtle case of $U$-controlled intrinsic frustration where the stiffness can be tuned to zero at finite $U$. Another common consequence of frustration is the AF band broadening, which is due to same-sublattice hopping $t^{\prime}$ in the square lattice and due to the spiral ordering in the triangular lattice. The consequent reduction of the AF band gap also enhances the fluctuation correction due to interband transfer of spectral weight. Furthermore, the suppression of the perfect-nesting instability in both cases has the result that magnetic ordering sets in only above a critical interaction strength. Whether the onset of magnetic ordering and opening of AF band gap occur at different $U$ values, yielding an intervening AFM phase for the triangualr lattice, is a pertinent question. At the HF level, an AFM phase is indeed stabilized, although in a narrow $U$ range $4.7<U<5.1$, indicating that the magnetic transition is nearly first order; whereas for the square lattice with $t^{\prime} / t \lesssim 0.5$, the magnetic order collapses when the two AF bands start overlapping, yielding a strictly first-order magnetic transition, the AFM phase is again stabilized for the cubic lattice 9.31 .32 This shows a subtle dependence on the density of overlapping band states, indicating that density of states renormalization due to quantum fluctuations should have an important effect on stabilization of the AFM phase.

We consider the Hubbard model

$$
\mathcal{H}=-t \sum_{i, \delta} a_{i, \sigma}^{\dagger} a_{i+\delta, \sigma}+U \sum_{i} a_{i \uparrow}^{\dagger} a_{i \uparrow} a_{i \downarrow}^{\dagger} a_{i \downarrow}
$$

with nearest-neighbour (NN) hopping on a triangular lattice, and focus on the effect of finite $U$-induced competing interactions on quantum spin fluctuations in the $120^{\circ}$ ordered state. The mean-field state is briefly reviewed in section II to introduce the notation. We obtain the spin fluctuation propagator (section III) and study the spinwave excitations and spin stiffness in the full $U$ range (section IV). We also examine the effects of hole and electron doping on magnetic ordering (section V).

\section{MEAN-FIELD STATE}

There are two alternative mean-field descriptions of the $120^{\circ}$ ordered AF state - i) a spiral-state representation, with an ordering wavevector $\mathbf{Q}=(2 \pi / 3,2 \pi / \sqrt{3})$, and ii) a three-sublattice representation, involving the local mean fields $\boldsymbol{\Delta}_{\alpha}$ on the three sublattices $\alpha=\mathrm{A}, \mathrm{B}, \mathrm{C}$. The energy eigenvalues and eigenvectors of the sublatticebasis $[6 \times 6]$ Hamiltonian matrix can be conveniently obtained from those of the spiral-state $[2 \times 2]$ Hamiltonian, as described below. 


\section{A. Spiral-state representation}

With an order parameter $\Delta_{\mathbf{Q}}=-U \sum_{\mathbf{k}}\left\langle a_{\mathbf{k}-\mathbf{Q} \downarrow}^{\dagger} a_{\mathbf{k} \uparrow}\right\rangle$, representing spin ordering in the $x-y$ plane, the Hubbard Hamiltonian reduces to

$$
H_{\mathrm{HF}}=\sum_{\mathbf{k}}\left(a_{\mathbf{k} \uparrow}^{\dagger} a_{\mathbf{k}-\mathbf{Q} \downarrow}^{\dagger}\right)\left[\begin{array}{cc}
\epsilon_{\mathbf{k}} & \Delta_{\mathbf{Q}} \\
\Delta_{\mathbf{Q}}^{*} & \epsilon_{\mathbf{k}-\mathbf{Q}}
\end{array}\right]\left(\begin{array}{l}
a_{\mathbf{k} \uparrow} \\
a_{\mathbf{k}-\mathbf{Q} \downarrow}
\end{array}\right)
$$

at the Hartree-Fock (HF) level, where $\epsilon_{\mathbf{k}}=-2 t\left[\cos k_{x}+\right.$ $\left.2 \cos \left(k_{x} / 2\right) \cos \left(k_{y} \sqrt{3} / 2\right)\right]$ is the triangular-lattice freefermion energy. Choosing real $\Delta_{\mathbf{Q}}$, the spiral-state quasiparticle energies $E_{\mathbf{k}}^{ \pm}$and amplitudes $\left(u_{\mathbf{k}} v_{\mathbf{k}}\right)$ are given by

$$
\begin{aligned}
E_{\mathbf{k}}^{ \pm} & =\eta_{\mathbf{k}} \pm \sqrt{\Delta_{\mathbf{Q}}^{2}+\xi_{\mathbf{k}}^{2}} \\
u_{\mathbf{k}}^{2} & =\frac{1}{2}\left(1 \pm \frac{\xi_{\mathbf{k}}}{\sqrt{\Delta_{\mathbf{Q}}^{2}+\xi_{\mathbf{k}}^{2}}}\right) \\
v_{\mathbf{k}}^{2} & =\frac{1}{2}\left(1 \mp \frac{\xi_{\mathbf{k}}}{\sqrt{\Delta_{\mathbf{Q}}^{2}+\xi_{\mathbf{k}}^{2}}}\right)
\end{aligned}
$$

for the upper $(+)$ and lower $(-)$ AF bands, where $\eta_{\mathbf{k}} \equiv$ $\left(\epsilon_{\mathbf{k}}+\epsilon_{\mathbf{k}-\mathbf{Q}}\right) / 2$ and $\xi_{\mathbf{k}} \equiv\left(\epsilon_{\mathbf{k}}-\epsilon_{\mathbf{k}-\mathbf{Q}}\right) / 2$. Self-consistency requires that $\Delta_{\mathbf{Q}}=-U \sum_{\mathbf{k}} v_{\mathbf{k}}^{*} u_{\mathbf{k}}$, which yields the condition

$$
\frac{1}{U}=\sum_{\mathbf{k}} \frac{1}{2 \sqrt{\Delta_{\mathbf{Q}}^{2}+\xi_{\mathbf{k}}^{2}}}\left[\theta\left(E_{\mathrm{F}}-E_{\mathrm{k}}^{-}\right)-\theta\left(E_{\mathrm{F}}-E_{\mathrm{k}}^{+}\right)\right]
$$

in which lower and upper band states contribute with opposite sign.

In terms of the fermion spinor $\Psi_{i}=\left(a_{i \uparrow} a_{i \downarrow}\right)$, the spin expectation values $\left\langle S_{i}^{\mu}\right\rangle=\frac{1}{2}\left\langle\Psi_{i}^{\dagger} \sigma^{\mu} \Psi_{i}\right\rangle$ in the spiral state yield

$$
\begin{aligned}
\left\langle S_{i}^{x}\right\rangle & =\frac{1}{2} m_{\mathbf{Q}} \cos \mathbf{Q} \cdot \mathbf{r}_{\mathbf{i}} \\
\left\langle S_{i}^{y}\right\rangle & =\frac{1}{2} m_{\mathbf{Q}} \sin \mathbf{Q} \cdot \mathbf{r}_{\mathbf{i}}
\end{aligned}
$$

at lattice site $i$, where the spiral-state magnetization $m_{\mathbf{Q}}=2 \sum_{\mathbf{k}}\left\langle a_{\mathbf{k}-\mathbf{Q} \downarrow}^{\dagger} a_{\mathbf{k} \uparrow}\right\rangle$. For $\mathbf{Q}=(2 \pi / 3,2 \pi / \sqrt{3})$ the spiral twisting of spins generates the $120^{\circ}$ ordered AF state on the triangular lattice.

\section{B. Three-sublattice representation}

While the spiral-state description applies only to Bravais lattices, the sublattice-basis description applies to Kagomé type non-Bravais lattices as well. In the HartreeFock approximation, the Hamiltonian reduces to

$$
\mathcal{H}_{\mathrm{HF}}=\sum_{i} \Psi_{i}^{\dagger}\left[-\boldsymbol{\sigma} \cdot \boldsymbol{\Delta}_{i}\right] \Psi_{i}-t \sum_{i, \delta} \Psi_{i}^{\dagger} \mathbf{1} \Psi_{i+\delta},
$$

where the local mean field $\boldsymbol{\Delta}_{i}=\frac{1}{2} U\left\langle\Psi_{i}^{\dagger} \boldsymbol{\sigma} \Psi_{i}\right\rangle$. In general, the $120^{\circ}$ AF state is characterized by an ordering plane (normal $\left.\hat{n}_{1}\right)$ and a planar direction $\left(\hat{n}_{2}\right)$ with reference to which $\boldsymbol{\Delta}_{i}$ make angles $\theta_{\alpha}=0^{0}, 120^{\circ}$, and $240^{\circ}$ on the three sublattices $\alpha=\mathrm{A}, \mathrm{B}, \mathrm{C}$. A convenient choice is $\hat{n}_{1}=\hat{z}$ (spin-ordering in the $x-y$ plane) and $\hat{n}_{2}=\hat{x}$, so that $\boldsymbol{\Delta}_{i}$ on the three sublattices are given by

$$
\boldsymbol{\Delta}_{\alpha}=\Delta \hat{\alpha} \quad(\hat{\alpha}=\hat{a}, \hat{b}, \hat{c})
$$

in terms of the three lattice unit vectors

$$
\hat{a}=\hat{x}, \quad \hat{b}=-\frac{1}{2} \hat{x}+\frac{\sqrt{3}}{2} \hat{y}, \quad \hat{c}=-\frac{1}{2} \hat{x}-\frac{\sqrt{3}}{2} \hat{y} .
$$

In general, in terms of the two mutually perpendicular directions $\hat{n}_{1}$ and $\hat{n}_{2}$, the spin orientations in Eq. (8) are given by

$$
\hat{\alpha}=\cos \theta_{\alpha} \hat{n}_{2}+\sin \theta_{\alpha}\left(\hat{n}_{1} \times \hat{n}_{2}\right)
$$

As $\boldsymbol{\Delta}_{i}$ is identical on sites of the same sublattice, Fourier transformation within the sublattice basis yields

$$
\mathcal{H}_{\mathrm{HF}}=\sum_{\mathbf{k}} \Psi_{\mathbf{k}}^{\dagger}\left[\begin{array}{ccc}
-\sigma \cdot \boldsymbol{\Delta}_{\mathrm{A}} & \delta_{\mathbf{k}} & \delta_{\mathbf{k}}^{*} \\
\delta_{\mathbf{k}}^{*} & -\sigma \cdot \boldsymbol{\Delta}_{\mathrm{B}} & \delta_{\mathbf{k}} \\
\delta_{\mathbf{k}} & \delta_{\mathbf{k}}^{*} & -\sigma \cdot \boldsymbol{\Delta}_{\mathrm{C}}
\end{array}\right] \Psi_{\mathbf{k}} .
$$

Here $\Psi_{\mathbf{k}} \equiv\left(a_{\mathbf{k} \uparrow} a_{\mathbf{k} \downarrow} b_{\mathbf{k} \uparrow} b_{\mathbf{k} \downarrow} c_{\mathbf{k} \uparrow} c_{\mathbf{k} \downarrow}\right)$, where $a_{\mathbf{k}}, b_{\mathbf{k}}, c_{\mathbf{k}}$ are fermion operators defined on the three sublattices A, B, C. Wavevector $\mathbf{k}$ lies within the Magnetic Brillouin Zone (MBZ), corresponding to the three inter-penetrating triangular sublattices (lattice parameter $\sqrt{3} a$ ). The NN hopping term

$$
\delta_{\mathbf{k}}=-t \sum_{\hat{\delta}=\hat{a}, \hat{b}, \hat{c}} e^{i \mathbf{k} \cdot \hat{\delta}}=-t\left[e^{i k_{x}}+2 e^{-i k_{x} / 2} \cos \left(\sqrt{3} k_{y} / 2\right)\right]
$$

mixes $\mathrm{AB}, \mathrm{BC}$, and $\mathrm{CA}$ sublattices, which are connected by the three lattice unit vectors.

The six eigenvalues $E_{\mathbf{k}, l}^{ \pm}$and eigenvectors $\left|\mathbf{k}^{ \pm}, l\right\rangle$ of the $[6 \times 6]$ Hamiltonian matrix in Eq. (11), corresponding to upper $(+)$ and lower $(-)$ AF bands, follow from Eqs. (3) and (4) for the spiral-state Hamiltonian. Here $l=1,2,3$ refer to the three branches corresponding to momentum values $\mathbf{k}, \mathbf{k}+\mathbf{Q}, \mathbf{k}-\mathbf{Q}$, respectively, $\mathbf{k}$ being restricted to the MBZ. The amplitude $|\mathbf{k}, l\rangle_{\alpha}$ involves not only the spin orientation $\phi_{\alpha}$ corresponding to sublattice $\alpha$, but also a relative phase angle $\delta_{\mathbf{Q}}^{l, \alpha}$ associated with the spiral twisting, and is given by

$$
|\mathbf{k}, l\rangle_{\alpha}=\left(\begin{array}{l}
u_{\mathbf{k}, l} e^{-i \phi_{\alpha}} \\
v_{\mathbf{k}, l}
\end{array}\right) e^{i \delta_{\mathbf{Q}}^{l, \alpha}}
$$

where the planar spin orientations $\phi_{\alpha}=0^{0}, 120^{\circ}, 240^{0}$ for the three sublattices. The spiral phase angle

$$
\delta_{\mathbf{Q}}^{l, \alpha}=0, \pm \mathbf{Q} \cdot \mathbf{R}_{\alpha \mathrm{A}}
$$


(relative to A) for $l=1,2,3$, where $\mathbf{R}_{\alpha \mathrm{A}}$ is the primitive lattice vector connecting sublattices $\alpha$ and $\mathrm{A}$. In view of above structure of state $|\mathbf{k}\rangle$, the self-consistency condition $m_{\alpha}^{\mu}=2 \Delta_{\alpha}^{\mu} / U=\sum_{\mathbf{k}, l}\left\langle\mathbf{k}, l\left|\sigma^{\mu}\right| \mathbf{k}, l\right\rangle_{\alpha}$ retains the same form as in Eq. (5).

\section{SPIN FLUCTUATIONS}

Associated with the spontaneous symmetry breaking of the continuous spin-rotation symmetry of the Hubbard model, there exist gapless transverse fluctuation modes or Goldstone modes, involving fluctuations locally normal to the symmetry-breaking directions. These low-energy collective excitations, studied earlier in the context of doped cuprates, 33 play an important role in determining several important physical properties in ordered systems, such as stability of the mean-field ordered state, quantum corrections to the order parameter and groundstate energy, temperature dependence of the order parameter in three dimensions, and renormalization of electron (hole) spectral function and AF band gap due to fermion-magnon scattering of mobile carriers in the magnetic background.

We consider the time-ordered spin fluctuation propagator, the Fourier transform of which is given in the sublattice basis as

$$
\begin{aligned}
{[\chi(\mathbf{q}, \omega)]_{\alpha \beta}^{\mu \nu} } & =i \int d t e^{i \omega\left(t-t^{\prime}\right)} \sum_{j} e^{i \mathbf{q} \cdot\left(\mathbf{r}_{i}-\mathbf{r}_{j}\right)} \\
& \times\left\langle\Psi_{\mathrm{G}} \mid \mathrm{T}\left[S_{i}^{\mu}(t) S_{j}^{\nu}\left(t^{\prime}\right)\right] \Psi_{\mathrm{G}}\right\rangle
\end{aligned}
$$

where $\alpha, \beta=\mathrm{A}, \mathrm{B}, \mathrm{C}$ are the sublattice indices and $\mu, \nu=$ $x, y, z$ are the spin directions. To leading order in an inverse-degeneracy expansion, 34 equivalent to summing over all bubble diagrams,

$$
[\chi(\mathbf{q}, \omega)]=\frac{\frac{1}{2}\left[\chi^{0}(\mathbf{q}, \omega)\right]}{\mathbf{1}-U\left[\chi^{0}(\mathbf{q}, \omega)\right]}
$$

where the bare particle-hole propagator $\left[\chi^{0}(\mathbf{q}, \omega)\right]$ is obtained by integrating out the fermions in the brokensymmetry state. For the half-filled insulating state, the added hole (particle) states lie in the lower (upper) Hubbard band, and we have

$\left[\chi^{0}(\mathbf{q}, \omega)\right]_{\alpha \beta}^{\mu \nu}=\frac{1}{2} \sum_{\mathbf{k} l m} \frac{\left\langle\sigma^{\mu}\right\rangle_{\alpha}^{-+}\left\langle\sigma^{\nu}\right\rangle_{\beta}^{-+*}}{E_{\mathbf{k}-\mathbf{q}}^{+}-E_{\mathbf{k}}^{-}+\omega}+\frac{\left\langle\sigma^{\mu}\right\rangle_{\alpha}^{+-}\left\langle\sigma^{\nu}\right\rangle_{\beta}^{+-*}}{E_{\mathbf{k}}^{+}-E_{\mathbf{k}-\mathbf{q}}^{-}-\omega}$,

where $\left\langle\sigma^{\mu}\right\rangle_{\alpha}^{-+}$denotes the particle-hole spin matrix element on the $\alpha$ sublattice

$$
\left\langle\sigma^{\mu}\right\rangle_{\alpha}^{-+} \equiv\left\langle\mathbf{k}-\mathbf{q}^{+}, m\left|\sigma^{\mu}\right| \mathbf{k}^{-}, l\right\rangle_{\alpha}
$$

The bare particle-hole propagator $\left[\chi^{0}(\mathbf{q}, \omega)\right]_{\alpha, \beta}^{\mu \nu}$ is a $[9 \times$ 9] Hermitian matrix in the three sublattice $(\alpha=\mathrm{A}, \mathrm{B}, \mathrm{C})$ and three spin $(\mu=x, y, z)$ basis, the (real) eigenvalues

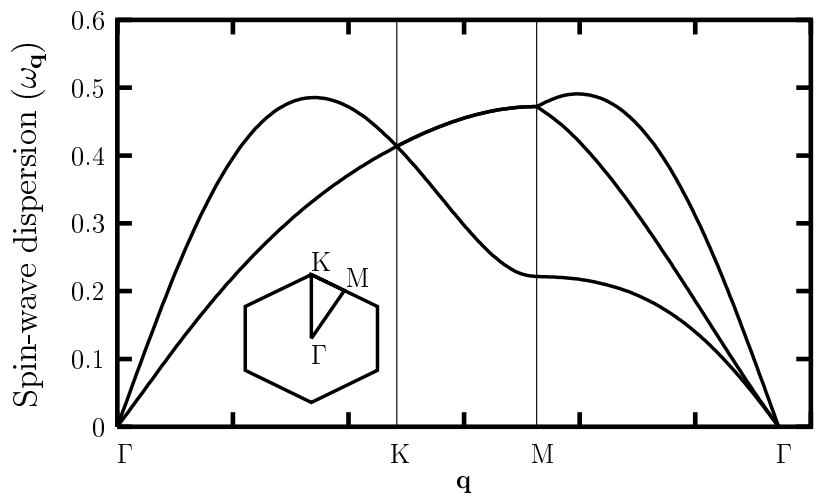

FIG. 1: The spin-wave dispersion along different symmetry directions of the MBZ (inset), for $\Delta=4(U=8.8)$.

$\lambda_{\mathbf{q}}(\omega)$ and eigenvectors $\left|\phi_{\mathbf{q}}(\omega)\right\rangle$ of which contain information regarding the massless transverse fluctuations (spin waves) as well as the massive longitudinal fluctuations along the local ordering direction. Also included are the particle-hole (Stoner) excitations across the band gap, given by the poles of $\left[\chi^{0}(\mathbf{q}, \omega)\right]_{\alpha, \beta}^{\mu \nu}$. In the following, we focus on the spin-wave energies $\omega_{\mathbf{q}}$, which are obtained from the poles $1-U \lambda_{\mathbf{q}}\left(\omega_{\mathbf{q}}\right)=0$ of Eq. (16).

Evaluating and diagonalizing the $\left[\chi^{0}(\mathbf{q}, \omega)\right]$ matrix for $\mathbf{q}, \omega=0$, we obtain three Goldstone modes $U \lambda_{n}=1$, as expected for the non-colinear $120^{\circ} \mathrm{AF}$ state, corresponding to rigid spin rotations about the $x, y, z$ axes. Rotation around the $z$ axis yields an in-plane mode involving $S^{x}$ and $S^{y}$ fluctuations, while those around the $x$ and $y$ axes yield two out-of-plane modes involving only $S^{z}$ fluctuation. The corresponding eigenvectors, giving transverse fluctuation amplitudes on the three sublattices, are

$$
\left(\begin{array}{r}
\hat{y} \\
-\frac{\sqrt{3}}{2} \hat{x}-\frac{1}{2} \hat{y} \\
\frac{\sqrt{3}}{2} \hat{x}-\frac{1}{2} \hat{y}
\end{array}\right) \quad\left(\begin{array}{r}
0 \\
\hat{z} \\
-\hat{z}
\end{array}\right) \text { and }\left(\begin{array}{r}
1 \\
\frac{1}{2} \hat{z} \\
-\frac{1}{2} \hat{z}
\end{array}\right)
$$

For small $\mathbf{q}, \omega$, the Goldstone mode eigenvalue $\lambda_{\mathbf{q}}(\omega)$ has the following typical form for the AF state:

$$
\lambda_{\mathbf{q}}(\omega)=\frac{1}{U}-\mathcal{A} q^{2}+\mathcal{B} \omega^{2},
$$

where the coefficient $\mathcal{A}$ of the $q^{2}$ term is proportional to the spin stiffness $\rho$. The pole equation $1-U \lambda=0$ yields the spin-wave energy $\omega_{\mathbf{q}}=c q$, where the spinwave velocity $c=\sqrt{\mathcal{A} / \mathcal{B}} \propto \sqrt{\rho}$ is related to the spin stiffness.

\section{SPIN-WAVE SPECTRUM}

The full spin-wave dispersion along different symmetry directions in the Magnetic Brillouin Zone is shown in Fig. 1 for $U=8.8$, corresponding to a self-consistent meanfield $\Delta=4$. Along the $\Gamma-\mathrm{K}$ direction, the softer mode is doubly degenerate, and the dispersion continues along 


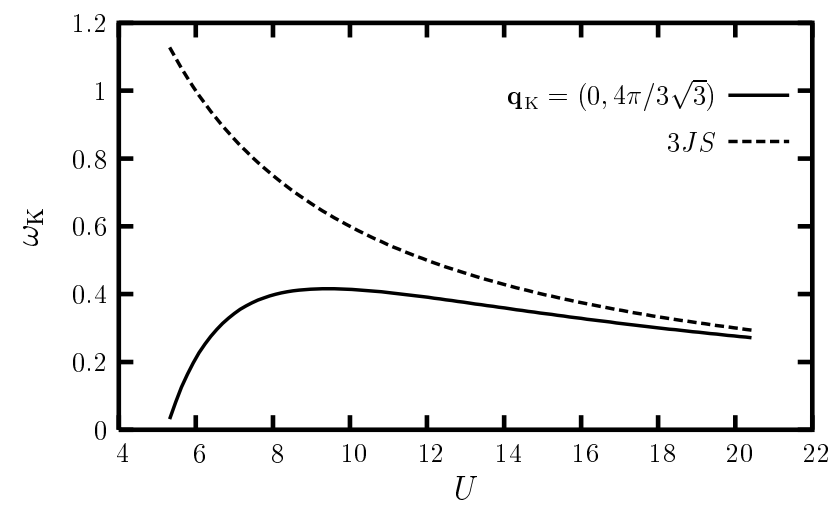

FIG. 2: The spin-wave energy $\omega_{K}$ at the Magnetic Brillouin Zone vertices $\mathrm{K}$, along with the strong-coupling result $3 J S$.

the $\mathrm{K}-\mathrm{M}$ direction after crossing at the $\mathrm{MBZ}$ vertex $\mathrm{K}$, where all three modes become degenerate. The degeneracies along $\Gamma-\mathrm{K}$ and $\mathrm{K}-\mathrm{M}$ directions in $\mathbf{k}$ space are simply related to the two equivalent $120^{\circ}$ orderings (clockwise and anticlockwise rotation of spins) along the corresponding orthogonal lattice directions $\hat{x}$ and $\frac{1}{2} \hat{x}+\frac{\sqrt{3}}{2} \hat{y}$. The degeneracy is resolved along the $\mathrm{M}-\Gamma$ direction, but two modes again become degenerate near $\Gamma$, and these represent the two out-of-plane $\left(S_{z}\right)$ fluctuation modes. Near the $\Gamma$ point $(q<<1)$, the $z$ sector does not mix with the $x-y$ sector in $\left[\chi^{0}(\mathbf{q}, \omega)\right]$, and the sublattice symmetry in $z$ sector yields the two degenerate $S_{z}$ fluctuation modes given in Eq. (19). Behaviour of $\omega_{\mathbf{q}}$ near the special MBZ points $\Gamma, \mathrm{K}$, and $\mathrm{M}$ is further discussed below.

We next consider the spin-wave energy at the MBZ vertices $K$ with $\mathbf{q}_{K}=(0,4 \pi / 3 \sqrt{3})$ etc., where all three spin-wave modes are degenerate, allowing for a convenient comparison with the strong-coupling result. In the strong-coupling limit $(U / t \rightarrow \infty)$, the spin-wave energies are given by:

$$
\omega_{\mathbf{q}}=3 J S\left[\left(1-\gamma_{\mathbf{q}}\right)\left(1+2 \gamma_{\mathbf{q}}\right)\right]^{1 / 2}
$$

where $\gamma_{\mathbf{q}}=\frac{1}{3}\left[\cos q_{x}+2 \cos \left(q_{x} / 2\right) \cos \left(q_{y} \sqrt{3} / 2\right)\right]$, and the three modes correspond to momenta $\mathbf{q}, \mathbf{q}+\mathbf{Q}$, and $\mathbf{q}-\mathbf{Q}$. As $\gamma_{\mathbf{q}}=0$ for all three modes for $\mathbf{q}_{K}=$ $(0,4 \pi / 3 \sqrt{3})$, the spin-wave modes are three-fold degenerate, with $\omega_{K}=3 J S=6 t^{2} / U$ for $S=1 / 2$. Figure 2 shows the variation of $\omega_{K}$ with $U$, along with the strongcoupling result for comparison. With decreasing $U$, the spin-wave energy turns over, and the decreasing band gap effectively squeezes the spin-wave spectrum. Indeed, both $\omega_{K}$ and the band gap vanish together at $U \approx 5$ $(\Delta=2)$. This is a typical weak-coupling dynamical effect for an itinerant antiferromagnet 35 The divergence in the eigenvalue $\lambda_{\mathbf{q}}(\omega)$ of the $\left[\chi^{0}(\mathbf{q}, \omega)\right]$ matrix in Eq. (17), when spin-wave energy approaches the band gap, not only limits the spin-wave spectrum to within the band gap, but also strongly suppresses the spin-wave amplitude due to wave-function renormalization.

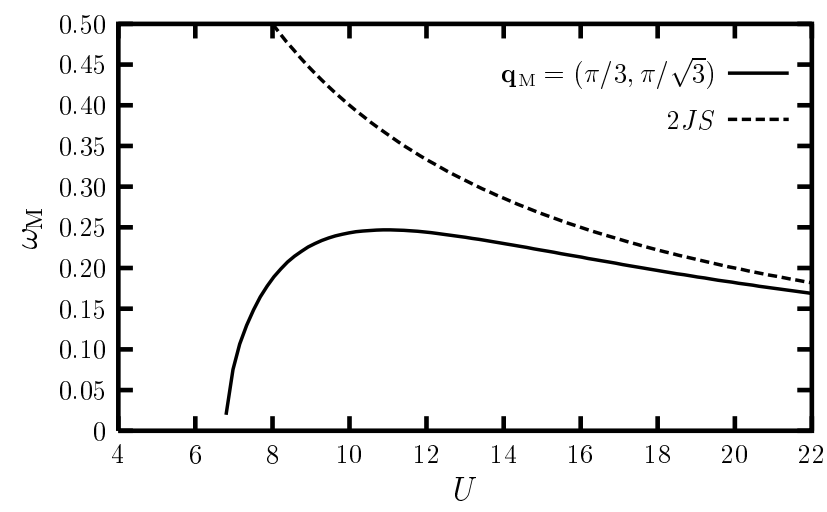

FIG. 3: The spin-wave energy $\omega_{M}$ at the mid-points (M) of the MBZ edges, along with the strong-coupling result $2 J S$.

While the spin-wave energy $\omega_{\mathrm{K}}$ at the MBZ vertices $\mathrm{K}$ vanishes along with the gap at $U \approx 5$, the spin-wave energy $\omega_{M}$ at the mid-points (M) of the MBZ edges shows evidence of competing interactions. Figure 3 shows the behaviour of $\omega_{\mathrm{M}}$ with $U$ for the soft, non-degenerate mode (see Fig. 1), along with the strong-coupling result $2 J S$. With decreasing $U, \omega_{\mathrm{M}}$ falls rapidly and vanishes at $U_{M}^{*}=6.8$. For $U<U_{M}^{*}$, the maximum eigenvalue of $\left[\chi^{0}\left(\mathbf{q}_{\mathrm{M}}\right)\right]$ exceeds $1 / U$, signalling an instability of the $120^{\circ}$ ordered state. This instability is driven by out-of-plane fluctuations, as the corresponding instability eigenvector $\phi_{\alpha}^{\mu}$ has non-vanishing amplitudes only for spin direction $\mu=z$, with identical magnitudes for all three sublattices $\alpha=A, B, C$. Analysis of the fluctuation amplitudes in the instability eigenvector indicates spin twisting in the $+z$ and $-z$ directions along alternating spin chains in the three lattice directions, implying instability towards a F-AF state, as in the square-lattice AF with NNN hopping $t^{\prime}$.

The spin-wave dispersion $\omega_{\mathbf{q}}$ along the K-M-K direction [Fig. 4] shows a cross-over from a quadratic behaviour around $\mathrm{M}$ for $U>U_{M}^{*}$ to a linear behaviour as $U \rightarrow U_{M}^{*}=6.8$. For $U<U_{M}^{*}$, there are no spin-wave solutions near $\mathrm{M}$ as $\lambda_{\mathbf{q}}>1 / U$. Despite the instability at $U=U_{M}^{*}$, the fluctuation remains finite as $U \rightarrow U_{M}^{*}$. This is because the magnon dispersion $\omega_{\mathbf{q}}$ near $\mathrm{M}$ becomes linear in (small) momentum difference $\tilde{\mathbf{q}} \equiv \mathbf{q}-\mathbf{q}_{M}$, and the fluctuation contribution of these modes $\left(\int \tilde{q} d \tilde{q} / c \tilde{q}\right)$ remains finite, just as for the Goldstone modes. A discontinuous magnetic transition at $U=U_{M}^{*}$ is therefore clearly indicated.

Turning now to long-wavelength modes, we consider the two spin-wave velocities $\left(c=\omega_{\mathbf{q}} / q\right)$ corresponding to in-plane $\left(c_{\|}\right)$and out-of-plane $\left(c_{\perp}\right)$ fluctuations. For $\mathbf{q}=0$, the off-diagonal matrix elements of $\left[\chi^{0}(\mathbf{q}, \omega)\right]$, involving in-plane $(\mu=x, y)$ and out-of-plane $(\nu=z)$ spin indices, vanish identically, implying no mixing between the in-plane mode of the $x-y$ sector and the two outof-plane modes of the $z$ sector. Mixing is negligible for $q<<1$ as well, and therefore small- $q$ spin-wave modes 


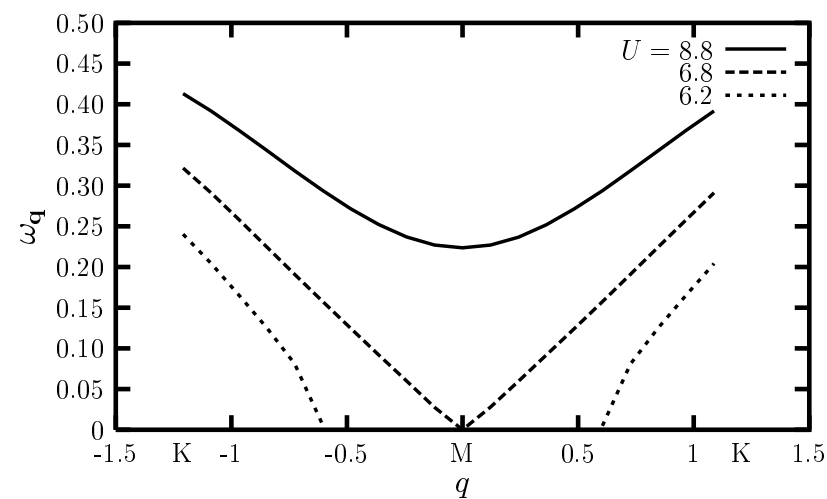

FIG. 4: The spin-wave dispersion $\omega_{\mathbf{q}}$ along the K-M-K direction, showing the cross-over from quadratic to linear behaviour around $\mathrm{M}$ as $U \rightarrow U_{M}^{*}=6.8$.

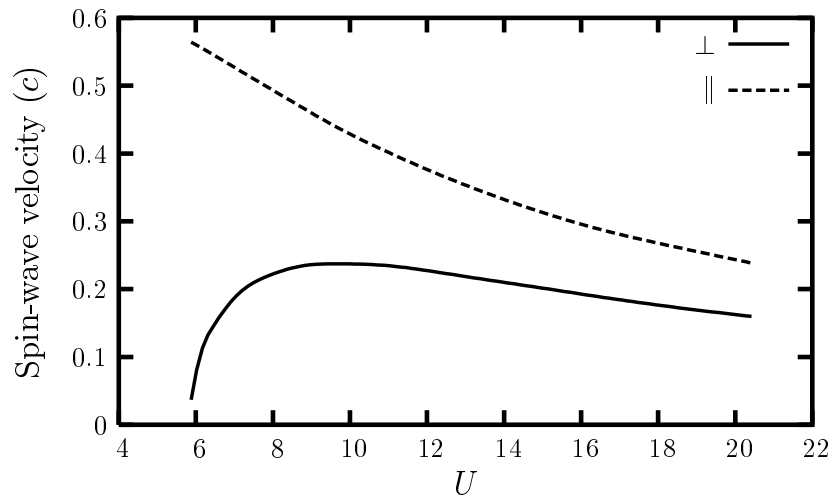

FIG. 5: The spin-wave velocities for in-plane $(\|)$ and outof-plane $(\perp)$ fluctuation modes, showing that $c_{\perp}$ vanishes at $U \approx 6$. The ratio approaches $\sqrt{2}$ in the strong coupling limit.

can also be identified in terms of in-plane and two outof-plane fluctuations. In view of the exact degeneracy along the $\Gamma-K$ direction, it is convenient to consider $\mathbf{q}=\left(0, q_{y}\right)$, as the two spin-wave energies then readily yield the two spin-wave velocities, as described below in the strong-coupling limit.

For $q_{x}=0$, the two modes $\mathbf{q} \pm \mathbf{Q}$ yield identical values: $\gamma_{\mathbf{q}}=-\frac{1}{3}\left[1 / 2+\cos \left(q_{y} \sqrt{3} / 2\right)\right]$, and hence degenerate spinwave energies from Eq. (21). For small $q_{y}$, one obtains $\omega_{\mathbf{q}}=3 J S(\sqrt{3} / 2) q_{y}$ and $3 J S(\sqrt{3} / 2 \sqrt{2}) q_{y}$ for the modes $\mathbf{q}$ and $\mathbf{q} \pm \mathbf{Q}$, respectively, yielding spin-wave velocities $c_{\|}=3 J S(\sqrt{3} / 2)$ and $c_{\perp}=3 J S(\sqrt{3} / 2 \sqrt{2})$, which are in the ratio $c_{\|} / c_{\perp}=\sqrt{2}$.

Figure 5 shows the two spin-wave velocities in the full $U$ range. Both velocities decrease as $1 / U$ for large $U$, and the ratio $c_{\|} / c_{\perp}$ asymptotically approaches $\sqrt{2}$ in the strong-coupling limit. The intermediate- $U$ behaviour for the spin-wave velocity corresponding to out-of-plane fluctuations is most interesting, which exhibits a broad peak and falls rapidly with decreasing $U$, vanishing at a critical interaction strength $U_{\text {stiff }}^{*} \approx 6$, which is in the insulating

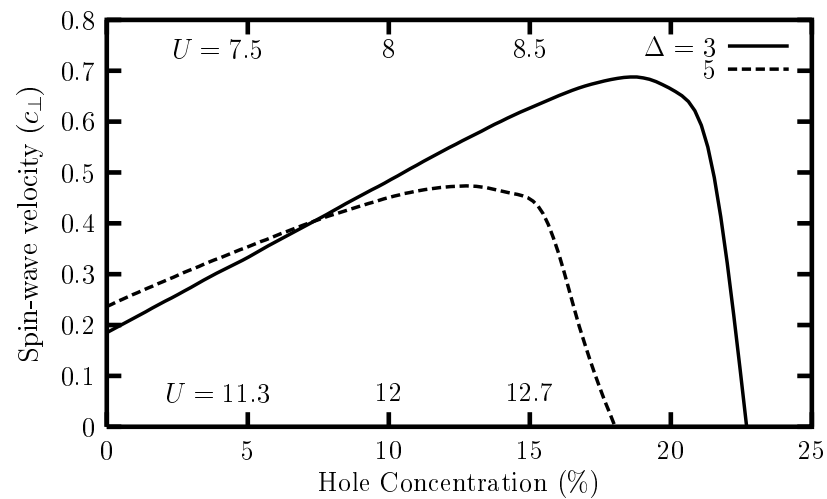

FIG. 6: Enhancement in $c_{\perp}$ with hole doping, due to a suppression of the intrinsic frustration in the triangular lattice.

regime. For $U<U_{\text {stiff }}^{*}$, the $120^{0} \mathrm{AF}$ state is therefore unstable with respect to out-of-plane fluctuations.

The vanishing spin-wave velocity and spin stiffness $\left(\rho_{\perp} \propto c_{\perp}^{2}\right)$ implies that the first-order quantum correction to sublattice magnetization due to transverse spin fluctuations diverges. Therefore, the corrected sublattice magnetization will vanish at a somewhat higher critical interaction strength $U_{\text {order }}^{*} \gtrsim U_{\text {stiff }}^{*}$, where the quantum reduction due to fluctuations exactly eliminates the mean-field order. Hence there is a quantum phase transition in the triangular-lattice $\mathrm{AF}$ at $U=U_{\text {order }}^{*}$ from a $120^{0}$ ordered state to a spin-disordered state. As described below, this QPT is driven by finite $U$-induced competing interactions and frustration.

In the strong-coupling limit, the Hubbard model at half filling maps to the $S=1 / 2 \mathrm{QHAF}$ with NN interactions. However, for finite $U$, extended-range spin couplings (of order $t^{4} / U^{3}$ and higher) are generated. Within the RPA analysis, the spin couplings are approximately given by $J_{i j} \approx U^{2}\left[\chi^{0}(\omega=0)\right]_{i j}$, and have been obtained for the square lattice from a systematic $t / U$ expansion ${ }^{36}$ Now, for the unfrustrated AF, the $\mathrm{O}\left(t^{4} / U^{3}\right)$ couplings between sites separated by two hoppings (NNN and NNNN) are ferromagnetic, which actually stabilize the AF state against the $t^{\prime}$-induced frustration ${ }^{9}$ However, for the $120^{\circ}$ state on the triangular lattice, the extended-range ferromagnetic couplings are a source of additional frustration, leading to spin softening.

\section{HOLE AND ELECTRON DOPING}

The two AF bands in the $120^{\circ}$ state are quite asymmetrical, with very different Fermi surfaces for hole and electron doping, suggesting quite different behaviour. Indeed, we find that while hole doping stabilizes the AF state and actually increases the spin stiffness, any electron doping destroys AF ordering.

Stability of the square-lattice AF state for hole and electron doping was studied earlier within the $t-t^{\prime}$ - 


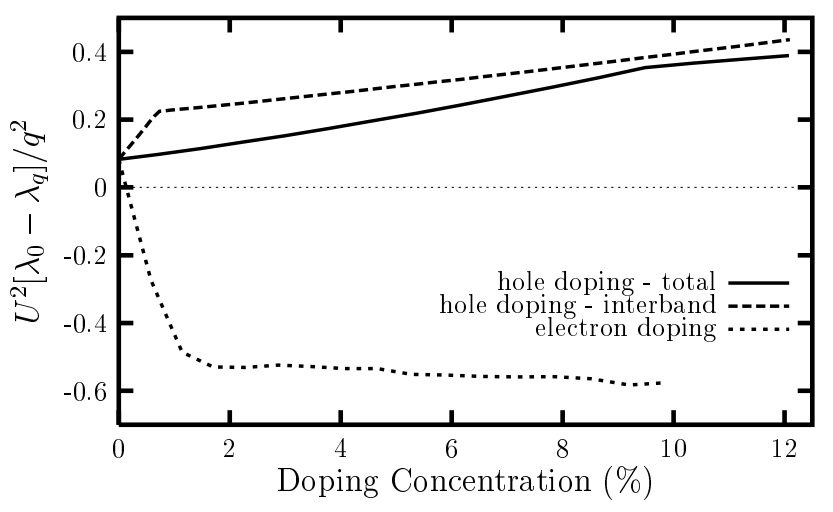

FIG. 7: Spin-stiffness contributions corresponding to total (interband+intraband) and interband particle-hole processes, for $\Delta=3$. The spin stiffness increases with hole doping (solid) whereas it becomes negative for electron doping (dotted).

Hubbard model 37 For finite doping, the Fermi energy lies within a band, and a key role is played by the intraband particle-hole processes in Eq. (17) for $\left[\chi^{0}(\mathbf{q}, \omega)\right]$, which generate additional frustrating spin couplings and affect the stability of the AF state with respect to transverse spin fluctuations. For positive $t^{\prime}$, the AF state is destroyed for any electron doping, while a finite concentration is required for hole doping.

We find a similar doping behaviour for the triangular lattice. For hole doping in the (broadened) lower AF band in a circular hole pocket around $\mathbf{k}=(0,0)$, the spinwave velocity $c_{\perp}$ initially increases (Fig. 6), and then rapidly falls to zero with further hole doping, indicating destruction of the AF state. The initial enhancement in $c_{\perp}$ is due to a suppression of the intrinsic frustration in the triangular lattice, as explained below. In order to highlight the role of doping, we have kept $\Delta$ rather than $U$ fixed, so that the band aspects remain unchanged with doping. Over a small doping range $U$ remains nearly same, as shown in the figure.

Figure 7 shows the spin-stiffness contributions corresponding to total (interband + intraband) and interband particle-hole processes in $\left[\chi^{0}(\mathbf{q}, \omega=0)\right]$. Here $\Delta=3$ and the $U$ values range between 7 and 8 . Interestingly, hole doping is seen to enhance the interband contribution, reflecting an effective suppression of the intrinsic frustration by eliminating the contribution of long-wavelength states in the lower AF band. While the intraband contribution (total - interband) does tend to destabilize the AF state (negative stiffness), the dominant interband contribution causes a net stabilization. A similar linear increase in the interband contribution with hole doping was obtained in a detailed analytical study of the $t-t^{\prime}$ Hubbard model 37

In contrast, for electron doping in the (narrow) upper $\mathrm{AF}$ band in elliptical electron pockets located symmetrically on the MBZ edges at $\mathbf{k}=\mathbf{Q} / 2=( \pm \pi / 3, \pm \pi / \sqrt{3})$ and $( \pm 2 \pi / 3,0)$, the spin stiffness abruptly turns nega- tive, indicating instability of the $120^{\circ} \mathrm{AF}$ state for any electron doping. Furthermore, the spin stiffness is seen to be quite independent of doping concentration. Very similar results for the $t-t^{\prime}$-Hubbard model were obtained for electron doping in the elliptical electron pockets around $\mathbf{k}=( \pm \pi / 2, \pm \pi / 2))^{37.38}$ The large relative magnitude of the intraband contribution is characteristic of the highly elliptical Fermi surface which reduces the energy denominator in the intraband particle-hole process.

\section{CONCLUSIONS}

In conclusion, spin-wave excitations were studied in the $120^{\circ}$ AF state of the Hubbard model on a triangular lattice at half filling as well as for electron and hole doping. The triangular-lattice antiferromagnet presents a novel case of $U$-controlled competing interactions and frustration, in contrast to the square-lattice case where frustration arises from the NNN coupling generated by the hopping term $t^{\prime}$. The spin-wave energy $\omega_{M}$ vanishes at $U_{M}^{*}=6.8$, and the instability of the $120^{\circ}$ ordered state against out-of-plane fluctuations implies a first-order quantum phase transition in the insulating state which pre-empts the vanishing spin-stiffness instability involving divergent quantum spin fluctuation and a continuous phase transition. The suppression of magnetic ordering due to enhanced quantum spin fluctuations arising from the finite $U$-induced frustration provides an explanation for why no long-range magnetic ordering is seen in the nearly isotropic triangularlattice antiferromagnet $\kappa-(\mathrm{BEDT}-\mathrm{TTF})_{2} \mathrm{Cu}_{2}(\mathrm{CN})_{3}$. Furthermore, the realization of a non-magnetic insulating state at intermediate $U$, which allows for a nonmagnetic insulator - paramagnetic metal transition when the AF band gap vanishes with decreasing $U$ and the two bands start overlapping, is relevant for the layered system $\kappa-(\mathrm{BEDT}-\mathrm{TTF})_{2} \mathrm{Cu}\left[\mathrm{N}(\mathrm{CN})_{2}\right] \mathrm{Cl}$, which exhibits a Mott-type metal-insulator transition not accompanied by any magnetic symmetry breaking.

A highly asymmetric doping behaviour was obtained for electron and hole doping, when the intraband particlehole processes were incorporated in the spin-fluctuation propagator. While hole doping was found to initially stabilize the AF state and actually increase the spin stiffness by suppressing the intrinsic frustration, any electron doping was found to destroy the $120^{\circ} \mathrm{AF}$ ordering. In fact, the stabilization for hole doping and instability for electron doping, with nearly concentration independent negative spin stiffness, closely resembles the doping behaviour of the square-lattice AF within the $t-t^{\prime}$-Hubbard model for positive $t^{\prime}$.

Finally, whether a non-magnetic insulator intervenes between the paramagnetic metal and the $\mathrm{AF}$ insulator depends on the relative strengths of the quantum corrections to magnetic order and $\mathrm{AF}$ band gap. In case the AF band gap closes before the magnetic order is lost, the magnetic transition is pre-empted, leaving a (nearly) 
first-order AFI - PM transition, when the AF bands start overlapping, possibly with an intervening AFM phase in a narrow $U$ range. In this context, quantum corrections to quasiparticle dispersion and band gap due to motion of an added hole (electron) in the $120^{\circ}$ ordered AF background are presently under investigation.

\section{Acknowledgement}

Helpful discussions with Z. Tešanović and V. Ashwin are gratefully acknowledged.
* Electronic address: avinas@iitk.ac.in

1 Y. Shimizu, K. Miyagawa, K. Kanoda, M. Maesato, and G. Saito, Phys. Rev. Lett. 91, 107001 (2003).

2 T. Komatsu, N. Matsukawa, T. Inoue, and G. Saito, J. Phys. Soc. Jpn. 65, 1340 (1996).

3 D. A. Huse and V. Elser, Phys. Rev. Lett. 60, 2531 (1988).

4 B. Bernu, P. Lecheminant, C. Lhuillier, and L. Pierre, Phys. Rev. B 50, 10048 (1994).

${ }^{5}$ L. Capriotti, A. E. Trumper, and S. Sorella, Phys. Rev. Lett. 82, 3899 (1999).

6 Th. Jolicoeur and J. C. Le Guillou, Phys. Rev. B 40, 2727 (1989).

7 A. V. Chubukov, S. Sachdev, and T. Senthil, J. Phys.: Condens. Matter 6, 8891 (1994).

8 P. Srivastava and A. Singh, Phys. Rev. B 70, 115103 (2004).

9 A. Singh, cond-mat/0112442 (2001).

10 R. R. P. Singh, Z. Weihong, C. J. Hamer, and J. Oitmaa, Phys. Rev. B 60, 7278 (1999).

11 V. N. Kotov, J. Oitmaa, O. Sushkov, and Z. Weihong, Phil. Mag. B 80, 1483 (2000).

12 L. Capriotti and S. Sorella, Phys. Rev. Lett. 84, 3173 (2000).

13 T. Senthil, S. Balents, S. Sachdev, A. Vishwanath, and M. P. A. Fisher, Phys. Rev. B 70, 144407 (2004).

14 F. Kagawa, T. Itou, K. Miyagawa, and K. Kanoda, Phys. Rev. B 69, 064511 (2004); F. Kagawa, T. Itou, K. Miyagawa, and K. Kanoda, cond-mat/0409437 (2004).

15 K. Kanoda, Physica C 282-287, 299 (1997); K. Kanoda, Hyperfine Interact. 104, 235 (1997).

16 R. H. McKenzie, Science, 278, 820 (1997).

17 K. Takada et al., Nature 422, 53 (2003).

18 H. H. Weitering, X. Shi, P. D. Johnson, J. Chen, N. J. Dinardo, and S. Kempa, Phys. Rev. Lett. 78, 1331 (1997).

19 G. Gasparovich, M. Kenzelmann, C. Broholm, L. N. Demianets, and A. Ya. Shapiro, (unpublished).
20 L. E. Svistov, A. I. Smirnov, L. A. Prozorova, O. A. Petrenko, L. N. Demianets, and A. Ya. Shapiro, Phys. Rev. B 67094434 (2003).

21 H. Morita, S. Watanabe, and M. Imada, J. Phys. Soc. Jpn. 71, 2109 (2002).

22 M. Capone, L. Capriotti, F. Becca, and S. Caprara, Phys. Rev. B 63, 085104 (2001).

${ }^{23}$ H. R. Krishnamurthy, C. Jayaprakash, S. Sarker, and W. Wenzel, Phys. Rev. Lett. 64, 950 (1990); C. Jayaprakash, H. R. Krishnamurthy, S. Sarker, and W. Wenzel, Europhys. Lett. 15, 625 (1991).

24 M. Fujita, T. Nakanishi, and K. Machida, Phys. Rev. B 45, 2190 (1992).

25 M. C. Refolio, J. M. López Sancho, and J. Rubio, cond-mat/0103459 (2001).

26 S.-W. Tsai and J. B. Marston, Can. J. Phys. 79, 1463 (2001).

27 R. Arita, K. Kuroki, and H. Aoki, J. Phys. Soc. Jpn. 73, 533 (2003).

28 Ph. Kurz, G. Bihlmayer, K. Hirai, and S. Blügel, Phys. Rev. Lett. 86, 1106 (2001).

29 T. Kashima and M. Imada, J. Phys. Soc. Jpn. 70, 3052 (2001).

30 M. Imada, T. Mizusaki, and S. Watanabe, cond-mat/0307022 (2003).

31 D. Duffy and A. Moreo, Phys. Rev. B 55, R676 (1997).

${ }^{32}$ W. Hofstetter and D. Vollhardt, Ann. Phys. (Leipzig) 7, 48 (1998)

33 A. Singh and Z. Tešanović, Phys. Rev. B 41, 614 (1990).

34 A. Singh, Phys. Rev. B 43, 3617 (1991).

35 P. Sen and A. Singh, Phys. Rev. B 48, 15792 (1993).

36 A. Singh, Phys. Rev. B 48, 6668 (1993).

37 A. Singh and H. Ghosh, Phys. Rev. B 65, 134414 (2002).

38 A. Singh, cond-mat/0207032 (2002). 